\title{
Staircase in mammalian muscle without light chain phosphorylation
}

D.E. Rassier,

L.A. Tubman and B.R. MacIntosh

\author{
Human Performance Laboratory and Department of Medical Science, \\ University of Calgary, Calgary, Alberta, Canada
}

\section{Correspondence \\ B.R. MacIntosh \\ Human Performance Laboratory \\ University of Calgary \\ Calgary, Alberta \\ Canada, T2N $1 \mathrm{~N} 4$ \\ Fax: (403) 284-3553 \\ E-mail: brian@ kin.ucalgary.ca}

Research supported by the $\mathrm{N}$ atural Science and Engineering Research Council of Canada. D.E. Rassier and $\mathrm{A}$. Tubman are recipients of CAPES and the Rick Hansen Man in Motion Foundation fellowships, respectively.

Received M ay 26, 1998 Accepted November 3, 1998

\section{Abstract}

In disuse atrophied skeletal muscle, the staircase response is virtually absent and light chain phosphorylation does not occur. The purpose of the present study was to determine if staircase could be restored in atrophied muscle with continued absence of myosin light chain phosphorylation, by reducing what appears to be an otherwise enhanced calcium release. Control (untreated) and sham-operated female Sprague-Dawley rats were compared with animals after 2 weeks of complete inactivity induced by tetrodotoxin (TTX) application to the left sciatic nerve. In situ isometric contractile responses of rat gastrocnemius muscle were analyzed before and after administration of dantrolene sodium (DS), a drug which is known to inhibit $\mathrm{Ca}^{2+}$ release in skeletal muscle. Twitch active force (AF) was attenuated by DS from $2.2 \pm 0.2 \mathrm{~N}, 2.7 \pm 0.1 \mathrm{~N}$ and $2.4 \pm 0.2 \mathrm{~N}$ to $0.77 \pm 0.2 \mathrm{~N}, 1.05 \pm$ $0.1 \mathrm{~N}$ and $1.01 \pm 0.2 \mathrm{~N}$ in TTX $(\mathrm{N}=5)$, sham $(\mathrm{N}=11)$ and control $(\mathrm{N}$ =7) muscles, respectively. Following dantrolene treatment, $10 \mathrm{~s}$ of 10 $\mathrm{Hz}$ stimulation increased AF to $1.32 \pm 0.2 \mathrm{~N}, 1.52 \pm 0.1 \mathrm{~N}$ and $1.45 \pm$ $0.2 \mathrm{~N}$ for the TTX, sham and control groups, respectively, demonstrating a positive staircase response. Regulatory light chain (R-LC) phosphorylation was lower for TTX-treated $(5.5 \pm 5.5 \%)$ than for control $(26.1 \pm 5.3 \%)$ and sham $(20.0 \pm 5 \%)$ groups. There was no significant change from resting levels for any of the groups after DS treatment $(\mathrm{P}=0.88)$. This study shows that treatment with dantrolene permits staircase in atrophied muscle as well as control muscle, by a mechanism which appears to be independent of R-LC phosphorylation.

\section{Introduction}

Staircase potentiation is a progressive enhancement of twitch active force in fasttwitch mammalian skeletal muscle that occurs during the early phase of repetitive low frequency stimulation $(1,2)$. This is a fundamental contractile property of muscle for which a mechanism has not been fully elucidated. Most evidence suggests that the mechanism by which twitch potentiation occurs is
Key words

- Potentiation

- $\mathrm{Ca}^{2+}$ availability

- Tetrodotoxin (TTX)

- Atrophy

- Myosin light chains 
use atrophy, TTX is delivered continuously to the sciatic nerve for a period of two weeks. Since TTX is a sodium channel blocker, all nerve action potential propagation is stopped, and the affected muscles are completely quiescent during the period of application (6). It has also been shown that R-LC phosphorylation is significantly lower in TTX-disuse atrophied muscle after $10 \mathrm{~s}$ of $10-\mathrm{Hz}$ repetitive stimulation in comparison with control or sham-treated muscles (8), suggesting that the direct relation between R-LC phosphorylation and staircase response is maintained in this model.

Muscle atrophy is also associated with an increase in the volume fraction of the sarcoplasmic reticulum (9), and the radial diffusion distances for $\mathrm{Ca}^{2+}$ within each myofibril must be shorter in atrophied muscle than in untreated muscle. These morphological changes would be expected to alter the $\mathrm{Ca}^{2+}$ concentration and distribution in the sarcoplasm (10-12), changing the contractile responses of atrophied muscle. These changes include a higher twitch:tetanus ratio (6) and a higher twitch:double-pulse contraction ratio in atrophied muscle when compared to non-atrophied muscle (5). A double-pulse contraction represents a very brief tetanic contraction and the twitch:double-pulse ratio reflects very well the same properties as the twitch:tetanic ratio without the risk of incurring fatigue due to excessive stimulation. Activation of the contractile proteins appears to be more complete with a single pulse in atrophied muscle than in non-atrophied muscle. This limits the range over which potentiation mechanisms can effectively contribute to enhancement of the twitch response.

In a previous paper (13) we have shown that staircase could be restored under conditions which limit the initial release of $\mathrm{Ca}^{2+}$ from the sarcoplasmic reticulum, thereby decreasing the twitch:tetanus ratio. That was accomplished by using dantrolene sodium, a specific inhibitor of sarcoplasmic reticulum
$\mathrm{Ca}^{2+}$ release (14-16). The purpose of this study was to investigate if staircase potentiation could be restored in atrophied muscle after dantrolene treatment without concomitant increases in R-LC phosphorylation. This would imply that factors other than R-LC phosphorylation are involved in twitch potentiation in skeletal muscle.

\section{Material and Methods}

Female Sprague-Dawley rats weighing approximately $200 \mathrm{~g}$ were used for this study. Rats were housed in a room with a $12-\mathrm{h}$ light-dark cycle, and standard rat chow and water were provided ad libitum. Care and treatment of these animals were consistent with the policies of the Canadian Council on Animal Care and were approved by a committee for the ethical use of animals for research.

Rats were randomly assigned to one of three groups: 1) a control group (no chronic treatment) $(\mathrm{N}=7), 2)$ a TTX-treated group $(\mathrm{N}=7)$ and 3$)$ a sham-treated group $(\mathrm{N}=14)$. Some animals originally from the TTX- and sham-treated groups were excluded from the study, following analysis of the twitch: double-pulse contraction ratio values. These exclusions were made due to apparent nerve damage in 3 of the 14 sham-treated animals and apparent failure of TTX delivery in 2 of the 7 animals in that group. Based on previous reports of change in the twitch:doublepulse contraction ratio (5), a twitch:doublepulse contraction ratio higher than 0.57 was accepted for TTX-treated animals, and a value lower than 0.57 was accepted for the sham-treated animals. Assurance that the remaining TTX-treated animals did not suffer nerve damage was evident in the recovery of nerve function following removal of the osmotic pump (see below).

Animals in the TTX-treated group had an osmotic pump (Alzet mini-osmotic pump, model 2002) implanted subcutaneously on the back while under pentobarbital sodium 
$(50 \mathrm{mg} / \mathrm{kg})$ anesthesia. A polyethylene tube was used to connect the osmotic pump to a silastic cuff placed around the left sciatic nerve. The osmotic pump was loaded with TTX (300-350 mg/ml) in sterile physiological saline containing 10,000 units of penicillin and $10 \mathrm{mg}$ of streptomycin/ml, and the delivery tube was primed with the same solution. After the surgery, incisions were sutured and each animal was returned to the cage and to initial conditions (free access to rat chow and water) for the next two weeks. Animals in the sham-treated group underwent the same surgical procedures as the animals in the TTX-treated group, but the osmotic pump and connecting tube were loaded only with sterile saline containing penicillin and streptomycin.

Fourteen days after the surgical implant, the osmotic pumps were removed, and $48 \mathrm{~h}$ later contractile characteristics were assessed. At this time, the rats in the TTX-treated group were expected to show a reversal of the acute effects of TTX treatment, permitting indirect activation of the gastrocnemius muscle.

\section{Muscle preparation}

Rats were deeply anesthetized with pentobarbital sodium $(50 \mathrm{mg} / \mathrm{kg}$ ip $)$ or ketaminexylazine $(1 \mathrm{ml} / \mathrm{kg}$; ketamine $100 \mathrm{mg} / \mathrm{ml}$, xylazine $100 \mathrm{mg} / \mathrm{ml}$, mixed 85:15 im). A deep level of anesthesia was maintained throughout the procedures with supplemental injections when needed. Once a suitable level of anesthesia had been obtained, a cannula (PE60) was placed into a common jugular vein. This cannula was used for administration of dantrolene sodium or propylene glycol. Dantrolene sodium was prepared for injection by dissolving in propylene glycol $(40 \mathrm{mg} / \mathrm{ml})$.

The left hindlimb was shaved on the caudal surface and an incision was made through the skin and superficial fascia from the calcaneus to the dorsal midline. The superficial muscles of the hindlimb were cut to expose the triceps surae, and the gastrocnemius muscle was cleared of connective tissue. Insertions of the soleus and plantaris muscles were detached from the Achilles tendon and were partly separated from the gastrocnemius muscle, and the calcaneus was cut. At this time, the plantaris muscle was frozen for subsequent biochemical analysis. The Achilles tendon was connected to an isometric force transducer (Grass Instruments FT10 with black/blue springs) with an inextensible wire. The hindlimb was immobilized with two pins: one placed perpendicularly into the femur and the other axially into the severed tibia. The sciatic nerve was isolated from adjacent connective tissue and severed close to the spine. The distal stump of the cut sciatic nerve was placed across a pair of stainless steel wire electrodes for indirect stimulation of the gastrocnemius muscle. The loosened skin from the hindlimb was stretched and anchored to form a pocket for warmed mineral oil. Rectal and mineral oil temperatures were monitored and regulated at approximately $37^{\circ} \mathrm{C}$ throughout the experiment using radiant heat.

\section{Experimental procedures}

Stimulation was with supramaximal (1$10 \mathrm{~V}$ ) square pulses, $50 \mu \mathrm{s}$ in duration (Grass Model S88). After testing contractions at a variety of lengths using paired pulses with a 5-ms delay, the muscle was set at the length which gave the strongest paired-pulse contraction (optimal length). In each experiment, there were three periods of assessment of the contractile response: i) control, ii) after vehicle injection, and iii) after dantrolene injection. Each period of contractile assessment included four twitch contractions at 20 -s intervals, a paired pulse contraction (5-ms delay) and a sequence of repetitive stimulation $(10 \mathrm{~Hz}$ for $10 \mathrm{~s})$. The twitch contractions were elicited to ensure consistent twitch active force in the gastrocnemius muscle. The paired pulse contraction was 
elicited to measure the twitch:double-pulse contraction ratio, and the repetitive stimulation was used to determine the extent and time-course of staircase. The muscle was allowed to rest for $5 \mathrm{~min}$ after the $10-\mathrm{Hz}$ stimulation before subsequent treatment. This rest interval was intended to prevent fatigue. Propylene glycol (vehicle alone) was administered intravenously 6 min before the second sequence of repetitive stimulation, and dantrolene sodium was administered before the third sequence of repetitive stimulation. The volume of dantrolene solution injected was approximately $0.3 \mathrm{ml} / \mathrm{kg}$, which was sufficient to reduce twitch active force to less than $50 \%$ of the pretreatment active force. After injections of propylene glycol or dantrolene, four twitch contractions at regular intervals and a paired pulse contraction were elicited as described above. These contractions permitted the assessment of the influence of propylene glycol and dantrolene sodium on the twitch and double-pulse contractions. The repetitive stimulation protocol $(10 \mathrm{~Hz}$ for $10 \mathrm{~s})$ was then performed, following the same procedures as the first time. As quickly as possible after the last repetitive stimulation protocol, the gastrocnemius muscle was frozen for subsequent biochemical analysis.

The tension responses were monitored throughout the experimental procedures, and contractions were sampled by computer at intervals during the stimulation at the following times: during the control period (frequency $=0.05 \mathrm{~Hz}$ ), and during the repetitive stimulation sequences at 0,5 and $10 \mathrm{~s}$ of $10-\mathrm{Hz}$ stimulation. The digitized (4000-Hz sampling frequency) contractions were analyzed by computer to determine the active force (AF) of the twitches. AF was measured as the difference in tension between the baseline (initial tension) and the peak of the contraction.

\section{Myosin light chain phosphorylation}

The frozen muscle samples were ana- lyzed to determine the proportion of phosphorylated R-LC. Besides the gastrocnemius muscle, some samples of the plantaris muscle were analyzed to assess resting values for $\mathrm{R}$ LC phosphorylation, since this muscle was expected to undergo the same effects of TTX treatment as the gastrocnemius muscle. Also, this muscle is composed primarily of fast twitch fibers, like the gastrocnemius muscle, and therefore comparisons between these muscles are justified. The procedure used for analysis of R-LC phosphorylation was as described previously $(8,17-19)$. The muscle was homogenized and centrifuged, and the supernatant was subjected to pyrophosphate polyacrylamide gel electrophoresis to isolate the myosin. The band representing myosin was identified with Coomassie blue stain, cut from the gel, denatured and subjected to isoelectric focusing over a $\mathrm{pH}$ range of 4.5 to 6. Gels from isoelectric focusing were fixed, silver stained and then scanned for quantification using an LKB 2202 Ultroscan laser densitometer.

\section{Statistical analysis}

For every experimental condition (no treatment, propylene glycol and dantrolene sodium) the groups (TTX-treated, shamtreated and control) were analyzed for AF. A two-way analysis of variance (groups, treatment) with repeated measures (treatment) was used. To evaluate changes in the twitch:double-pulse contraction ratio for each group (control, sham-treated and TTXtreated), a one-way analysis of variance with repeated measures was used. To detect if the twitch:double-pulse contraction ratio was different in the TTX-treated group after dantrolene sodium treatment from the shamtreated and control groups in normal conditions (no treatment), a one-way analysis of variance was used. A two-way analysis of variance without replication was performed to detect interactions and/or differences in R-LC phosphorylation levels among the three 
groups (TTX-treated, sham-treated and control) following dantrolene sodium administration and between the two different conditions (resting condition, i.e., plantaris, and post-stimulation condition, i.e., gastrocnemius muscle). Multiple comparisons were done with the Newman-Keuls test. A significance level of $\mathrm{P}<0.05$ was used for detecting significant differences. Recognizing that the design of this study predicts "no significant difference" as an important observation (anticipating no change in R-LC phosphorylation in atrophied and dantrolene-treated atrophied muscle, or alternatively, no change in active force during $10-\mathrm{Hz}$ stimulation), a $P$ value $>0.2$ was used in these cases to avoid a type II error.

\section{Results}

\section{Single twitch contraction}

To determine the effects of propylene glycol and dantrolene sodium on the AF of the twitch, single twitch contractions were analyzed prior to any treatment and after the two injections. The results revealed no significant group:condition interaction $(\mathrm{P}=$ 0.67). Also, there were no significant group effects $(\mathrm{P}=0.21)$. Dantrolene sodium significantly $(\mathrm{P}<0.001)$ reduced $\mathrm{AF}$ in all groups (see Figure 1).

\section{Twitch:double-pulse contraction ratio}

The twitch:double-pulse contraction ratio gives an indication of the level of activation achieved with the first stimulus, relative to the level achieved with two (high frequency) pulses of activation (i.e., a very brief tetanic contraction). When the twitch: double-pulse contraction ratio is less than 0.5 it indicates that the second stimulation of a paired pulse stimulation elicited more tension development than the first pulse of the pair. In Table 1 it can be seen that the twitch:double-pulse ratio was less than 0.5 in all cases except the TTX-treated muscles prior to dantrolene treatment. A one-way analysis of variance within each group revealed that propylene glycol had no effect on this ratio, but dantrolene sodium treatment reduced the twitch:double-pulse contraction ratio in all groups $(\mathrm{P}<0.005)$. The intent of the dantrolene treatment was to permit a relatively normal contractile response in otherwise atrophied muscle. The twitch:doublepulse contraction ratio in the TTX-group after dantrolene treatment was similar to that expected in a non-atrophied muscle. In fact, in the TTX-group, the reduction in the ratio gave a value which was significantly smaller $(P=0.003)$ than this ratio in sham-treated and control groups prior to any treatment. A smaller dose of dantrolene may have resulted in a twitch:tetanus ratio not different from the control ratio.

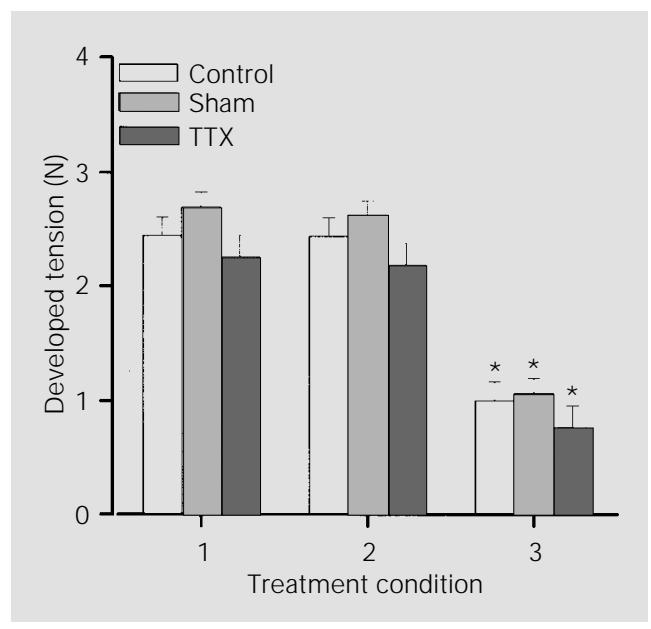

Figure 1 - Active force of twitch contractions in different conditions during the experiment. In this case, conditions are: 1) no treatment, 2) after propylene glycol treatment and 3) after dantrolene sodium treatment. Values are reported as means \pm SEM (vertical bars). Asterisks show that condition 3 for each group is significantly different from conditions 1 and 2 within the corresponding group $(P<0.001)$. TTX, Tetrodotoxin.
Table 1 - Twitch:double-pulse contraction ratio.

Values are reported as mean $\pm \mathrm{SEM} . *$ Significantly different from the other conditions, within a group $(\mathrm{P}<0.05)$. $\Pi \mathrm{x}$, Tetrodotoxin.

\begin{tabular}{llll}
\hline & \multicolumn{3}{c}{ Twitch:double-pulse contraction ratio } \\
\cline { 2 - 4 } & Control & Sham-treated & TTX-treated \\
\hline No treatment & $0.47 \pm 0.02$ & $0.46 \pm 0.01$ & $0.67 \pm 0.03$ \\
Propylene glycol & $0.47 \pm 0.02$ & $0.45 \pm 0.01$ & $0.64 \pm 0.03$ \\
Dantrolene sodium & $0.39 \pm 0.04^{*}$ & $0.38 \pm 0.01^{*}$ & $0.42 \pm 0.06^{*}$
\end{tabular}


Figure 2 - Active force vs time for $10 \mathrm{~s}$ of repetitive stimulation at $10 \mathrm{~Hz}$, after administration of propylene glycol (A) and dantrolene sodium (B). Values are reported as means \pm SEM (vertical bars). Asterisks at a given time depict a significant difference $(P<0.05)$ between groups at that time. See text for significant changes within each group. TTX, Tetrodotoxin.

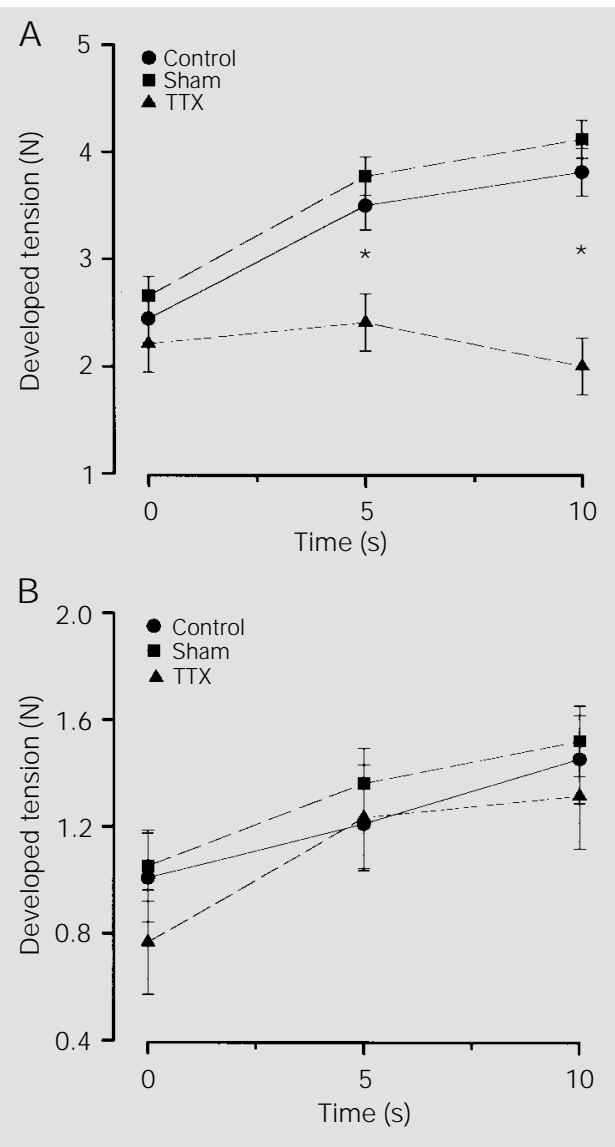

Active force during repetitive stimulation

As expected (2), $10 \mathrm{~s}$ of repetitive stimulation at $10 \mathrm{~Hz}$ resulted in a progressive staircase response in the non-atrophied muscles before and after (Figure 2A) injection of propylene glycol. A significant group by condition interaction was observed $(\mathrm{P}<0.005)$ for active force. In the control and sham-treated groups, the values for AF at $5 \mathrm{~s}$ and $10 \mathrm{~s}$ were significantly higher than they were with the first pulse of stimulation $(0 \mathrm{~s})$. Figure $2 \mathrm{~A}$ shows that, in contrast to the other groups, AF in the TTX-treated group did not change significantly from the value for the first contraction during the repetitive stimulation. The values for AF were significantly different between the TTX-treated group and the other groups at $5 \mathrm{~s}$ and $10 \mathrm{~s}$ (Figure 2A). However, after administration of dantrolene sodium, there was no group by time interaction $(\mathrm{P}=0.62)$ and no significant group effect $(\mathrm{P}=0.64)$. AF increased significantly above the value for the first contraction at 5 $\mathrm{s}$ and $10 \mathrm{~s}$ in all groups, in a clear positive staircase response to repetitive stimulation (see Figure 2B).

\section{Myosin light chain phosphorylation}

Myosin R-LC phosphorylation was measured in resting plantaris muscle without dantrolene treatment, and in gastrocnemius muscles after dantrolene treatment plus $10 \mathrm{~s}$ of $10-\mathrm{Hz}$ repetitive stimulation (see Table 2). Two way analysis of variance showed no significant group (control, TTX-treated and sham-treated) by condition (resting and poststimulation) interaction $(\mathrm{P}=0.88)$. There was a significant group effect $(\mathrm{P}=0.04)$ and no significant condition effect $(\mathrm{P}=0.88)$. The lack of a condition effect reveals that stimulation at $10 \mathrm{~Hz}$ for $10 \mathrm{~s}$ did not result in significant elevation of R-LC phosphorylation above the resting value. The bottom line of Table 2 (combined values) presents the mean values for each group, regardless of condition (resting and stimulated), illustrating the significant group effect.

\section{Discussion}

In this study, it has been confirmed that staircase is absent in atrophied fast-twitch rat skeletal muscle. There are two novel observations reported in this study: 1) dantrolene treatment in atrophied muscle resulted in restoration of the staircase response without concomitant R-LC phosphorylation; 2) non-atrophied muscle also demonstrated a staircase response without corresponding R-LC phosphorylation after dantrolene treatment. These observations support the hypothesis that factors other than R-LC phosphorylation can contribute to staircase potentiation.

All results found in the sham-treated ani- 
mals in this study were similar to those found for the control animals. Therefore, any difference found between the TTX-treated group and the other groups is due to the TTXinduced atrophy and not to the surgical procedures.

The control and sham-treated groups presented a positive staircase response to $10 \mathrm{~s}$ of $10-\mathrm{Hz}$ stimulation prior to any injection. It is known that the staircase response is obtained by an increased intensity of activation, not a prolongation of activation $(14,20)$. In the control and sham animals prior to dantrolene treatment, R-LC phosphorylation probably plays an important role in the staircase response. Others have reported an increase in phosphorylation of R-LC to 0.6 $\mathrm{mol} / \mathrm{mol} \mathrm{R}-\mathrm{LC}$ after $10 \mathrm{~s}$ of $10-\mathrm{Hz}$ stimulation in association with a $75 \%$ increase in twitch active force $(17,21)$. The observations reported in this paper do not detract from the perceived importance of R-LC phosphorylation in effecting a staircase response $(21,22)$ under normal conditions.

The absence of potentiation in the TTXtreated animals before treatment with dantrolene sodium that has been reported previously (5-7) was confirmed in this study. This absence of potentiation is associated with an absence of R-LC phosphorylation (8), apparently confirming the importance of this process for twitch potentiation. It should be noted, however, that it is also possible that potentiation, which might otherwise have been seen in the atrophied muscles, was masked by early fatigue in these muscles. This would be very difficult to ascertain.

There is another factor that may affect the observation of potentiation in atrophied muscle. There is a high twitch:tetanus ratio (6) in these muscles, and the high twitch: double-pulse contraction ratio observed in our results and reported previously in the literature (5) confirms that there is a high relative level of activation which is apparently achieved with a single pulse of activation in atrophied muscle. This enhanced level
Table 2 - Myosin regulatory light chain phosphorylation.

aUnits are: mol of phosphate per mol of R-LC (means \pm SEM); bvalues presented for the plantaris muscle represent resting values; ${ }^{c}$ values presented for the gastrocnemius muscle represent poststimulated condition; dnumbers in parentheses represent number of samples for that condition. *Significant group effect $(\mathrm{P}=$ 0.04), i.e., R-LC phosphorylation of TTX-treated muscles is lower than control or sham-treated muscles regardless of history of stimulation. TTx, Tetrodotoxin.

\begin{tabular}{llll}
\hline & \multicolumn{3}{c}{ Myosin regulatory light chain phosphorylationa } \\
\cline { 2 - 4 } & \multicolumn{1}{c}{ Control } & Sham-treated & TTX-treated \\
\hline Plantaris $^{b}$ & $26.8 \pm 2.8(3)^{\mathrm{d}}$ & $20.6 \pm 1.9(3)$ & $2.8 \pm 2.8(3)$ \\
Gastrocnemius $^{\mathrm{c}}$ & $25.3 \pm 6.0(7)$ & $19.4 \pm 4.1(10)$ & $8.2 \pm 3.8(5)$ \\
Combined & $26.1 \pm 5.3(10)$ & $20.0 \pm 5.0(13)$ & $5.5 \pm 5.5^{*}(8)$
\end{tabular}

of activation may decrease the opportunity for potentiation. This is consistent with the lack of staircase in atrophied muscle. Also, this could explain why a staircase response was observed after administration of dantrolene sodium in these muscles. Dantrolene would be expected to decrease the level of activation per stimulating pulse, and this would increase the available change in active force which can occur, and would perhaps also decrease the impact of fatigue in these muscles, which may otherwise be superimposed on a potentiation response. However, for staircase to occur in these atrophied muscles, it would have to be by some mechanism other than R-LC phosphorylation, since there is no change in the level of phosphorylation from the apparent resting condition (see Table 2).

The resting values for R-LC phosphorylation are somewhat high in comparison to other published values (17), but not beyond the range reported in the literature (22). RLC phosphorylation levels in the control and sham-treated groups after $10 \mathrm{~s}$ of $10-\mathrm{Hz}$ stimulation were considerably less than what would be expected after this stimulation. This is not due to methodological concerns, since our laboratory has consistently observed values of R-LC phosphorylation of 
about $50 \%$ in stimulated muscle not treated with dantrolene $(8,19)$.

It has been previously reported that a greater R-LC phosphorylation level would be expected in non-atrophied muscles (not treated with dantrolene), i.e., $60 \%$ after $10 \mathrm{~s}$ of stimulation(21). Presumably the decreased levels of R-LC phosphorylation reported in the current study are due to attenuated $\mathrm{Ca}^{2+}$ release because of dantrolene treatment. A decreased level of $\mathrm{Ca}^{2+}$ liberated from the sarcoplasmic reticulum would be anticipated to reduce the quantity of the $\mathrm{Ca}^{2+}$-calmodulin complex available to bind with and activate myosin light chain kinase (MLCK). Similarly, activation of MLCK in the control and sham-treated muscles would also be reduced or prevented, resulting in no increase in phosphorylation of R-LC during the repetitive stimulation. Since staircase potentiation was observed in the three groups after dantrolene treatment, it would appear that under this condition (attenuated $\mathrm{Ca}^{2+}$ release) staircase potentiation is independent of R-LC phosphorylation. This is not unlike the situation in fatigued muscle, which undergoes substantial potentiation during $10-\mathrm{Hz}$ stimulation with very little R-LC phosphorylation (21).

The principal assumption in this paper is that dantrolene acts on the muscle by inhibition of $\mathrm{Ca}^{2+}$ release, and this mechanism of action has been confirmed (23). There are several studies which confirm this action of dantrolene and exclude other potential effects. It is known that this drug does not affect motor nerve function, neuromuscular transmission or muscle membrane function $(24,25)$. The reduced twitch amplitude and the absence of R-LC phosphorylation in the control muscles are further evidence that dantrolene effectively reduced $\mathrm{Ca}^{2+}$ release in these muscles. However, since $\mathrm{Ca}^{2+}$ concentrations were not measured in this study, this action of dantrolene cannot be directly confirmed.

The main purpose of this study was to investigate the contractile responses of atrophied muscle after administration of dantrolene sodium, to determine if a staircase response could be attained in the apparent absence of R-LC phosphorylation. Regardless of the actual mechanism by which dantrolene effected a reduction in twitch active force, the observation of a staircase response in these muscles as well as the control and sham-treated muscles without a concomitant increase in R-LC phosphorylation demonstrates that factors other than R-LC phosphorylation can contribute to a staircase response. This is a novel observation, which will undoubtedly trigger additional research in an effort to gain a better understanding of this fundamental contractile property of skeletal muscle.

\section{Acknowledgments}

The authors would like to thank Dr. Walter Herzog for his valuable comments during the preparation of this paper. 


\section{References}

1. Desmedt J E \& Hainaut K (1968). Kinetics of myofilament activation in potentiated contraction: staircase phenomenon in human skeletal muscle. Nature, 217: 529-532.

2. Maclntosh BR (1991). Skeletal muscle staircase response with fatigue or dantrolene sodium. Medicine and Science in Sports and Exercise, 23: 56-63.

3. Sweeney HL \& Stull JT (1986). Phosphorylation of myosin in permeabilized mammalian cardiac and skeletal muscle cells. American J ournal of Physiology, 250: C657-C660.

4. Persechini A, Stull J T \& Cooke R (1985). The effect of myosin phosphorylation on the contractile properties of skinned rabbit skeletal muscle fibers. J ournal of Biological Chemistry, 260: 7951-7954.

5. Maclntosh BR, Roberge MC \& Gardiner PF (1988). Absence of staircase following disuse in rat gastrocnemius muscle. $\mathrm{Ca}$ nadian J ournal of Physiology and Pharmacology, 66: 707-713.

6. St-Pierre DMM \& Gardiner PF (1985). Effect of "disuse" on mammalian fasttwitch muscle: joint fixation compared with neurally applied tetrodotoxin. Experimental Neurology, 90: 635-651.

7. St-Pierre DMM, Leonard D, Houle R \& Gardiner PF (1988). Recovery of muscle from tetrodotoxin-induced disuse and the influence of daily exercise. Experimental Neurology, 101: 327-346.

8. Tubman LA, MacIntosh BR \& Rassier DE (1996). Absence of myosin light chain phosphorylation and twitch potentiation in atrophied skeletal muscle. Canadian J ournal of Physiology and Pharmacology, 74: 723-728.
9. Engel AG \& Stonnington HH (1974). Morphological effects of denervation of muscle: a quantitative ultrastructural study. Annals of the New York Academy of Sciences, 228: 68-88.

10. Dulhunty AF \& Gage PW (1985). Excitation-contraction coupling and charge movement in denervated rat extensor digitorum longus and soleus muscles. J ournal of Physiology, 583: 75-89.

11. Dulhunty AF \& Gage PW (1983). Asymmetrical charge movement in slow- and fast-twitch mammalian muscle fibres in normal and paraplegic rats. Journal of Physiology, 341: 213-231.

12. Finol HJ, Lewis DM \& Owens R (1981). The effects of denervation on contractile properties of rat skeletal muscle. J ournal of Physiology, 319: 81-92.

13. Rassier DE, Tubman LA \& MacIntosh BR (1997). Inhibition of Ca2 + release in rat atrophied gastrocnemius muscle. Experimental Physiology, 82: 665-676.

14. Desmedt JE \& Hainaut K (1977). Inhibition of the intracellular release of calcium by dantrolene in barnacle giant muscle fibers. J ournal of Physiology, 265: 565-585.

15. Krarup C (1981). The effect of dantrolene on the enhancement and diminution of tension evoked by staircase and by tetanus in rat muscle. J ournal of Physiology, 311: 389-400.

16. Leslie GC \& Part NJ (1981). The action of dantrolene sodium on rat fast and slow muscle in vivo. British J oumal of Pharmacology, 72: 665-672.

17. Moore RL \& Stull JT (1984). Myosin light chain phosphorylation in fast and slow skeletal muscles in situ. American J our- nal of Physiology, 247: C462-C471.

18. Silver PJ \& Stull J T (1982). Quantitation of myosin light chain phosphorylation in small tissue samples. J ournal of Biological Chemistry, 257: 6137-6144.

19. Tubman LA, Maclntosh BR \& Maki WA (1996). Myosin light chain phosphorylation and posttetanic potentiation in fatigued skeletal muscle. Pflügers Archiv, 431: 882-887.

20. Krarup C (1981). Enhancement and diminution of mechanical tension evoked by staircase and by tetanus in rat muscle. J ournal of Physiology, 311: 355-372.

21. Maclntosh BR, Grange RW, Cory CR \& Houston ME (1993). Myosin light chain phosphorylation during staircase in fatigued skeletal muscle. Pflügers Archiv, 425: 9-15.

22. Klug GA, Biedermann $M$, Houston ME, Stuart D, Mumby M \& Stull JT (1992). Chronic low frequency stimulation reduces myosin phosphorylation in rabbit fast twitch muscle. Canadian J ournal of Physiology and Pharmacology, 70: 859-865.

23. Hainaut K \& Desmedt J E (1974). Effect of dantrolene sodium on calcium movements in single muscle fibres. Nature, 252: 728-730.

24. Ellis KO \& Carpenter J F (1972). Studies on the mechanism of action of dantrolene sodium. Naunyn-Schmiedeberg's Archives of Pharmacology, 275: 83-94.

25. Ellis KO \& Carpenter J F (1974). Mechanism of control of skeletal-muscle contraction by dantrolene sodium. Archives of Physical Medicine and Rehabilitation, 55: 362-369. 